\title{
EVALUATION OF SOME MEDICINAL PLANT EXTRACTS FOR THEIR NEMATICIDAL PROPERTIES AGAINST ROOT-KNOT NEMATODE, MELOIDOGYNE INCOGNITA
}

\author{
HUMAIRA $^{1 *}-$ SHAD, A. A. ${ }^{1}-$ MUHAMMAD, D. $^{2}-$ SHAH, H. U. ${ }^{1}$ \\ ${ }^{1}$ Department of Agricultural Chemistry, Faculty of Nutrition Sciences, The University of \\ Agriculture Peshawar, Pakistan \\ ${ }^{2}$ Department of Soil and Environmental Sciences, Faculty of Crop Production Sciences, The \\ University of Agriculture Peshawar, Pakistan \\ ${ }^{*}$ Corresponding author \\ e-mail:aminses@yahoo.com
}

(Received $9^{\text {th }}$ Aug 2018; accepted $31^{\text {st }}$ Oct 2019)

\begin{abstract}
Nematicidal activities of some plant extracts were assayed against Meloidogyne incognita.. Six different medicinal plants were collected from Swat valley of Pakistan. Plants were moderately washed with tap water in order to remove various impurities like dust, dirt and adhering materials. The plants were shade dried and powdered. Aqueous Methanolic extraction of the dried powdered samples were carried out through cold percolation technique followed by removal of the solvent using vacuum rotary evaporator under controlled temperature. The methanolic crude extracts were further fractionated using different polarity solvents n-hexane, chloroform, ethyl acetate, and n-butanol. Methanolic extracts were screened for egg hatchability and nematicidal activity against second stage juveniles of $M . j$ incognita. in the laboratory under the microscope. The nematode eggs and juveniles were exposed 24, 48 and $72 \mathrm{hrs}$ in different concentrations (10,100, $1000 \mathrm{ppm})$ of plant extracts. The plant extracts of Acacia modesta (roots), Segeratia thea (leaves), and Celtis caucasic (aerial part) exhibited highly promising mortality of more than $60 \%$ after $72 \mathrm{hrs}$ exposure. There was a gradual decrease in egg hatching with increase in extract concentration of Acacia modesta (roots), Segeratia thea (leaves), and Celtis caucasic (aerial part) were found to be the most effective in reducing egg hatching. Larval mortality were strongly influenced by concentration of extract, plant species and duration of period.
\end{abstract}

Keywords: Segeratia thea, methanolic extraction, larval mortality, Acacia modesta, juveniles

\section{Introduction}

Root-knot nematode, Meladogyne incognita. is one of the major plant- parasitic nematodes adversely affecting the quantity and quality of the major crops. Plant parasitic nematodes are the main pathogens on most food crops and without adequate control cause loss of yield and quality. Yield losses due to plant parasitic nematodes have been reported to be $\$ 100$ billion worldwide annually (Sasser and Freckman, 1987). Root-knot nematodes (Meloidogyne species) infect almost all types of plants and cause considerable damage (Adekunle and Akinlua, 2007). Root knot nematode larvae infect plant root causing the development of root knot galls that affect the plant's photosynthetic process and nutrient uptake (Eisenback and Triantaphyllous, 1991).

Nematodes are difficult to control because of their high rate of reproduction and wide host range, while females are capable of producing up to one thousand eggs/female (Natarajan et al., 2006). Plant-parasitic nematodes are documented as the causes of serious yield losses on a wide range of crops (Javad et al., 2006). Current study shows that $M$. incognita. is the species which cause serious problem in various agricultural crops. 


$$
-2476 \text { - }
$$

Although chemical nematicides hold major role in nematode control but it is expensive and is economically viable only for high value crops and create a potential hazard to the environment and human health (Tsay et al., 2004). This issue has stimulated research on nematode management through natural products with nematicidal activity such as root exudates, plant volatile compounds (Linford et al., 1938), endophytic bacteria (Vetrivelkalai et al., 2010) and plant extracts (Muniasamy et al., 2010; Pavaraj et al., 2010). A wide variety of plant species, representing 57 families have been shown to exhibit nematicidal compounds (Sukul, 1992), which includes isothiocynates, thiophenics glycosides, alkaloids, phenolics and fatty acids (Gommers, 1973). Nematicidal phytochemicals are generally safe for the human health and environment (Chitwood, 2002). Hence, the present study has been carried out to evaluate some plant extracts for their nematicidal properties against root-knot nematode M. incognita..

\section{Material and methods}

Several hundred plants wildly grow in Swat valley and adjacent areas. Due to their socioeconomic, agricultural and pharmacological potential they are very popular in the native people. Debregeasia saeneb aerial part (stem + leaves), Celtis caucasica aerial part (stem + leaves), Acacia modesta (roots \& bark), Segeretia thea (leaves), Isodon rugosus (roots), Buxus papillosa (leaves + stem + roots) were used in the study. The plants samples were collected from different regions of district Swat and district Upper Dir (Figure 1). The collected species were moderately washed with tap water to remove various impurities like dust, dirt and adhering materials. Then were shade dried until complete dryness of the plant material was achieved. The dried samples were grinded by using electrical grinder. The powdered samples were extracted in aqueous methanol followed by removal of the solvent using vacuum rotary evaporator under controlled temperature.

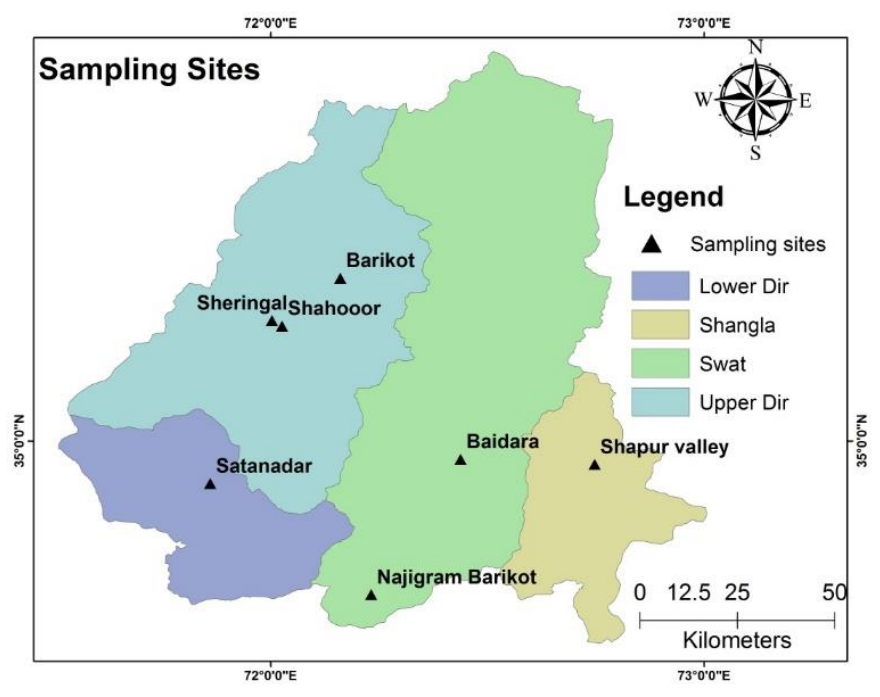

Figure 1. Map of the sampling sites

The methanolic crude extracts were further fractionated using different polarity solvents n-hexane, chloroform, ethyl acetate, and n-butanol in separating funnel. The 
crude extracts and their respective fractions were then dissolved in 5\% aqueous DMSO to prepare stock solution. Different concentrations of plant extracts $(10,100,1000 \mathrm{ppm})$ were prepared from the stock solution using 5\% aqueous DMSO. For obtaining of egg masses and larvae pure culture of $M$. incognita. were collected from the roots of infected Red duranta plant. Plant roots were washed well to remove soil and debris. Then infected roots were cut into $2-3 \mathrm{~cm}$ pieces. Root pieces were placed in a blender. Enough water was added to cover the roots and blended for $15-20 \mathrm{sec}$ at low speed. The suspension was poured through the sieves $25 \mu \mathrm{m}, 36 \mu \mathrm{m}, 63 \mu \mathrm{m}, 100 \mu \mathrm{m}$. The top sieve (100 $\mu \mathrm{m}$ mesh) was used to retain debris, washed with tap running water.

Eggs on the 500 mesh sieve are gentle washed under a slow stream of cold tap water. The eggs were collected from the 500 mesh sieve into a beaker. The egg suspension was brought to a known volume to determine the number of eggs per millilitre. $1 \mathrm{ml}$ of the egg suspension was used in counting plate with the plant extract and was kept at room temperature for 24,48 and $72 \mathrm{hrs}$ for egg hatchability test. The nematodes eggs were exposed to 10, 100 and 1000 ppm of each plant extract for 24, 48 and $72 \mathrm{hrs}$. Four plant extracts exhibited highly promising mortality rates of more than $60 \%$ after $72 \mathrm{hrs}$ of exposure $(\mathrm{P}<0.05)$.

Rest of the egg suspension was stored in saline solution in refrigerator. For the juveniles study the freshly extracted egg suspension was kept in incubator at $24^{\circ} \mathrm{C}$ for 4 days to let the eggs hatched and get the second stage juveniles. Effect on egg hatching was evaluated on mature uniform size eggs of $M$. incognita. were suspended in the extract, 5\% aqueous DMSO (control) and nemacur (nematicide) replicated three times in counting plates. The counting plates were kept at room temperature. Observations were recorded on number of eggs hatched after 24, 48, 72 hrs. For effect of \% mortality freshly hatched $\mathrm{J}_{2}$ of $M$. incognita. were placed in each dilutions and control, replicated three times in counting plates. The plates were kept at room temperature. Mortality of larvae was calculated as a percent of total larvae suspended (Cayrol et al., 1989).

$$
\begin{aligned}
& \% \text { egg hatching }=\text { No. of hatched eggs } / \text { Total No. of eggs X } 100 \\
& \% \text { Mortality }=\text { No. of dead juveniles / Total No. of juveniles X } 100
\end{aligned}
$$

\section{Statistical analysis}

All the data were analysed using 2-way factorial experimental design at 5\% level of significance, using statistical package Statistix. The letters showing $a, b$ and $c$ with the mean values shows that the means are significantly different from each other.

\section{Results and Discussions}

\section{\% Egg Hatchability of Plant Methanolic Crude Extracts}

A gradual decrease was observed in egg hatching with increase in plant extract concentration (Table 1). Abdalla et al. (2008) reported that methanol and hexane extracts of the 27 samples were screened for nematicidal activity against second stage juveniles of $M$. incognita in the laboratory. The present study revealed that plant extracts of Acacia modesta (roots), Segeratia thea (leaves), Isodon rugosus (roots), Celtis caucasica (arial part) were found to be most effective in reducing \% egg 
hatching. Plant extracts of Boxus papillosa (leaves, stem and roots), Acacia modesta (bark) and Debregeasea saeneb proved to be less effective against $M$. incognita.

Table 1. Effect of different concentrations of plant crude extracts on \%egg hatchability in the root-knot nematode, Meloidogyne incognita

\begin{tabular}{|c|c|c|c|c|}
\hline \multirow[t]{2}{*}{ Plants } & \multirow{2}{*}{$\begin{array}{l}\text { Exposure time } \\
\text { hours }\end{array}$} & \multicolumn{3}{|c|}{$\begin{array}{c}\% \text { of egg hatching at different dilutions (ppm) of plant } \\
\text { extract }\end{array}$} \\
\hline & & 10 & 100 & 1000 \\
\hline \multirow{4}{*}{ Acacia modesta (roots) } & \multirow{4}{*}{$\begin{array}{l}24 \\
48 \\
72\end{array}$} & 54.38 & 31.23 & 20.80 \\
\hline & & 51.36 & 20.10 & 14.53 \\
\hline & & 24.30 & 15.23 & 8.30 \\
\hline & & 43.35a & $22.19 b$ & $14.54 c$ \\
\hline \multirow{4}{*}{ Acacia modesta (bark) } & \multirow{4}{*}{$\begin{array}{l}24 \\
48 \\
72\end{array}$} & 43.73 & 27.76 & 22.2 \\
\hline & & 38.13 & 25.03 & 15.93 \\
\hline & & 29.80 & 22.20 & 8.30 \\
\hline & & 37.22a & $25.00 b$ & $15.54 \mathrm{c}$ \\
\hline \multirow{4}{*}{ Segeretia thea (leaves) } & \multirow{4}{*}{$\begin{array}{l}24 \\
48 \\
72\end{array}$} & 86.07 & 23.57 & 20.8 \\
\hline & & 18.03 & 11.07 & 8.30 \\
\hline & & 14.53 & 8.30 & 6.23 \\
\hline & & 39.54a & $14.31 b$ & $11.77 b$ \\
\hline \multirow{4}{*}{ Isodon rugosus (roots) } & \multirow{4}{*}{$\begin{array}{l}24 \\
48 \\
72\end{array}$} & 40.23 & 33.3 & 18.7 \\
\hline & & 33.3 & 25.66 & 14.53 \\
\hline & & 29.13 & 23.26 & 9.70 \\
\hline & & 34.22a & $27.41 b$ & 14.31c \\
\hline \multirow{4}{*}{ Buxus papillosa (leaves) } & \multirow{4}{*}{$\begin{array}{l}24 \\
48 \\
72\end{array}$} & 77.76 & 68.73 & 44.40 \\
\hline & & 61.76 & 49.30 & 27.76 \\
\hline & & 51.36 & 26.36 & 19.40 \\
\hline & & 63.63a & 48.13b & $30.52 \mathrm{c}$ \\
\hline \multirow{4}{*}{ Buxus papillosa (stem) } & \multirow{4}{*}{$\begin{array}{l}24 \\
48 \\
72\end{array}$} & 58.30 & 42.33 & 27.06 \\
\hline & & 52.76 & $\begin{array}{l}42.35 \\
37.46\end{array}$ & 25.66 \\
\hline & & 44.40 & 33.96 & 23.56 \\
\hline & & 51.82a & 37.92b & $25.43 c$ \\
\hline \multirow{4}{*}{ Buxus papillosa (roots) } & \multirow{4}{*}{$\begin{array}{l}24 \\
48 \\
72\end{array}$} & 88.16 & 77.06 & 67.96 \\
\hline & & 77.66 & 67.33 & 47.23 \\
\hline & & 66.63 & 54.83 & 39.50 \\
\hline & & $77.48 \mathrm{a}$ & $66.41 b$ & $51.56 \mathrm{c}$ \\
\hline \multirow{4}{*}{$\begin{array}{c}\text { Debregeasia saeneb (aerial } \\
\text { part) }\end{array}$} & \multirow{4}{*}{$\begin{array}{l}24 \\
48 \\
72\end{array}$} & 75.00 & 48.46 & 31.13 \\
\hline & & 63.16 & 38.13 & 21.50 \\
\hline & & 57.63 & 34.83 & 20.10 \\
\hline & & $65.26 a$ & $40.47 b$ & $24.24 c$ \\
\hline \multirow{4}{*}{ Celtis caucasica (aerial part) } & \multirow{4}{*}{$\begin{array}{l}24 \\
48 \\
72\end{array}$} & 44.43 & 38.86 & 29.13 \\
\hline & & 33.26 & 27.73 & 20.80 \\
\hline & & 24.96 & 17.33 & 10.36 \\
\hline & & $34.22 \mathrm{a}$ & $27.97 b$ & $20.10 b$ \\
\hline
\end{tabular}

\section{\% Mortality of Plants Methanolic Crude Extracts}

The methanolic crude extracts of Acacia modesta (Roots), Segeratia thea (leaves), Isodon rugosus (roots) and Celtis caucasica (aerial part) exhibited mortality more than $50 \%$ after 72 hrs exposures (Table 2). Pavaraj et al. (2012) observed the plant extracts effect on egg hatching of nematode and mortality of the second stage juveniles of M. incognita in vitro after 24, 48 and $72 \mathrm{hrs}$ of exposure. Similar results were observed in the current work from crude extracts of Segeratia thea (leaves) and Celtis caucasica (aerial part) exhibited 74.5 and $87.3 \%$ of larval mortality, while undiluted root extracts of Acacia modesta and Isodon rugosus exhibited 69 and 54\% of larval mortality each at $1000 \mathrm{ppm}$ concentration after $72 \mathrm{hrs}$ of exposure, respectively. Egg hatching inhibition 
and larval mortality decreased with decrease in the concentration of the extracts. Juvenile mortality increased parallel to an increased time of exposure. The potential of using plant extracts in controlling plant parasitic nematodes has been shown by several authors (Adegbite and Adesiyan, 2005; Opareke et al., 2005; Orisajo et al., 2007; Abbasi et al., 2008). Extracts from Segeratia thea and Celtis caucasica were the most toxic compared to other plant extracts (Table 2). The nematicidal effect of the tested crude extracts may probably be because of higher content of certain oxygenated compounds which are considered to possess lipophilic properties that allow them to dissolve the cytoplasmic membranes of nematode cells and their functional groups interfering with enzyme protein structure (Knoblock et al., 1989).

Table 2. Effect of different concentrations of plant Methanolic crude extracts on larval mortality in the root-knot nematode, Meloidogyne incognita

\begin{tabular}{|c|c|c|c|c|}
\hline \multirow[t]{2}{*}{ Plants } & \multirow[t]{2}{*}{ Exposure time hours } & \multicolumn{3}{|c|}{$\begin{array}{c}\text { \% mortality of juveniles at different concentration (ppm) of } \\
\text { plant extract }\end{array}$} \\
\hline & & 10 & 100 & 1000 \\
\hline \multirow{4}{*}{ Acacia modesta (roots) } & \multirow{4}{*}{$\begin{array}{l}24 \\
48 \\
72\end{array}$} & 2.1 & 16.49 & 28.48 \\
\hline & & 3.2 & 31.17 & 56.34 \\
\hline & & 7.31 & 53.46 & 69.43 \\
\hline & & $4.20 \mathrm{c}$ & $33.70 \mathrm{~b}$ & $51.42 \mathrm{a}$ \\
\hline \multirow{4}{*}{ Acacia modesta (bark) } & \multirow{4}{*}{$\begin{array}{l}24 \\
48 \\
72\end{array}$} & 11 & 6.95 & 16.70 \\
\hline & & 14 & 21 & 27 \\
\hline & & 21 & 33 & 41.33 \\
\hline & & $15.33 \mathrm{c}$ & $20.31 \mathrm{~b}$ & 28.34 a \\
\hline \multirow{4}{*}{ Segeretia thea (leaves) } & \multirow{4}{*}{$\begin{array}{l}24 \\
48 \\
72\end{array}$} & 2.33 & 21.1 & 47.55 \\
\hline & & 13.33 & 25.56 & 54.41 \\
\hline & & 17.45 & 34.61 & 74.54 \\
\hline & & $11.04 \mathrm{c}$ & 27.09 b & 58.83 a \\
\hline \multirow{4}{*}{ Isodon rugosus (roots) } & \multirow{4}{*}{$\begin{array}{l}24 \\
48 \\
72\end{array}$} & 3.2 & 6.89 & 13.37 \\
\hline & & 15.22 & 21 & 24.27 \\
\hline & & 31 & 44 & 54.11 \\
\hline & & $16.47 \mathrm{c}$ & 23.96 b & 30.58 a \\
\hline \multirow{4}{*}{ Buxus papillosa (leaves) } & \multirow{4}{*}{$\begin{array}{l}24 \\
48 \\
72\end{array}$} & 1.2 & 24.14 & 6.35 \\
\hline & & 7.52 & 23.11 & 26.55 \\
\hline & & 10.33 & 27.32 & 36.52 \\
\hline & & $6.35 \mathrm{~b}$ & 24.85 a & 23.14 a \\
\hline \multirow{4}{*}{ Buxus papillosa (stem) } & \multirow{4}{*}{$\begin{array}{l}24 \\
48 \\
72\end{array}$} & 1.3 & 11 & 13.16 \\
\hline & & 3.46 & 21 & 26.73 \\
\hline & & 13.6 & 31 & 46.36 \\
\hline & & $6.12 \mathrm{c}$ & $21 \mathrm{~b}$ & 28.75 a \\
\hline \multirow{4}{*}{ Buxus papillosa (roots) } & \multirow{4}{*}{$\begin{array}{l}24 \\
48 \\
72\end{array}$} & 4.36 & 7.58 & 24 \\
\hline & & 11 & 21.66 & 27 \\
\hline & & 15.22 & 35.44 & 51 \\
\hline & & $10.19 \mathrm{c}$ & $21.56 \mathrm{~b}$ & 34 a \\
\hline \multirow{4}{*}{$\begin{array}{c}\text { Debregeasia saeneb (aerial } \\
\text { part) }\end{array}$} & \multirow{4}{*}{$\begin{array}{l}24 \\
48 \\
72\end{array}$} & 3.33 & 11 & 23 \\
\hline & & 6.52 & 16.22 & 34 \\
\hline & & 12.1 & 25.22 & 44 \\
\hline & & $7.31 \mathrm{c}$ & $17.48 \mathrm{~b}$ & 33.66 a \\
\hline \multirow{4}{*}{ Celtis caucasica (aerial part) } & \multirow{4}{*}{$\begin{array}{l}24 \\
48 \\
72\end{array}$} & 2.4 & 21 & 41 \\
\hline & & 11 & 31.23 & 67.62 \\
\hline & & 21 & 41.54 & 87.37 \\
\hline & & $11.46 \mathrm{c}$ & $31.25 \mathrm{~b}$ & 65.33 a \\
\hline
\end{tabular}

In Acacia modesta (roots) among all the four fractions the chloroform and ethyl acetate fractions exhibited highly mortality rates of 68.13 and $70 \%$, respectively after 72 hours exposure (Table 3). In Segeretia thea (leaves) the chloroform and ethyl acetate 
fractions exhibit high mortality rates of 74.14 and $75.16 \%$, respectively after 72 hours exposure (Table 4). Celtis caucasica (aerial part) fractions caused larval mortality at the rate of n-hexane 84 , chloroform 86 , and ethyl acetate $88 \%$ after 72 hours of exposure at 1000 ppm (Table 5). While Isodon rugosus (roots) ethylacetate fraction showed highly mortality rates of juveniles at 55\% after 72 hours exposure time at $1000 \mathrm{ppm}$ concentration (Table 6). All the means regarding concentration level were significantly different among each plant extract. The nematicidal effect of ethyl acetate and chloroform fractions are may be due to the presence of nematicidal compounds isothiocynates, thiophenics glycosides, alkaloids, phenolics and fatty acids (Gommers, 1973).

Table 3. Effect of different concentrations of different polarity fractions of Acacia modesta (roots) on larval mortality of the root-knot nematode, Meloidogyne incognita

\begin{tabular}{|c|c|c|c|c|}
\hline \multirow[t]{2}{*}{ Acacia modesta (roots) } & \multirow[t]{2}{*}{ Exposure time hours } & \multicolumn{3}{|c|}{$\begin{array}{c}\% \text { mortality of juveniles at different dilutions (ppm) of } \\
\text { plant extract }\end{array}$} \\
\hline & & 10 & 100 & 1000 \\
\hline \multirow[t]{2}{*}{ n- hexane fraction } & \multirow[t]{2}{*}{$\begin{array}{l}24 \\
48 \\
72\end{array}$} & $\begin{array}{c}1.2 \\
2.13 \\
3.73 \\
\end{array}$ & $\begin{array}{c}13.6 \\
20.56 \\
39.96 \\
\end{array}$ & $\begin{array}{l}22.66 \\
42.43 \\
53.56 \\
\end{array}$ \\
\hline & & $2.35 c$ & $24.71 b$ & $39.55 a$ \\
\hline \multirow[t]{2}{*}{ Chloroform fraction } & \multirow[t]{2}{*}{$\begin{array}{l}24 \\
48 \\
72\end{array}$} & $\begin{array}{l}2.50 \\
3.60 \\
7.06 \\
\end{array}$ & $\begin{array}{l}15.93 \\
31.23 \\
42.96 \\
\end{array}$ & $\begin{array}{c}27.2 \\
55.23 \\
68.13 \\
\end{array}$ \\
\hline & & $4.40 \mathrm{c}$ & 30.04b & 50.18a \\
\hline \multirow[t]{2}{*}{ Ethyl acetate fraction } & \multirow[t]{2}{*}{$\begin{array}{l}24 \\
48 \\
72\end{array}$} & $\begin{array}{c}3.2 \\
4.3 \\
7.86 \\
\end{array}$ & $\begin{array}{c}17.13 \\
33.43 \\
54.6 \\
\end{array}$ & $\begin{array}{c}28.48 \\
57.4 \\
70.15 \\
\end{array}$ \\
\hline & & $5.12 \mathrm{c}$ & $35.05 b$ & $52.01 a$ \\
\hline \multirow[t]{2}{*}{ n- butanol fraction } & \multirow[t]{2}{*}{$\begin{array}{l}24 \\
48 \\
72\end{array}$} & $\begin{array}{l}1.63 \\
2.20 \\
5.63 \\
\end{array}$ & $\begin{array}{l}14.76 \\
30.28 \\
51.76 \\
\end{array}$ & $\begin{array}{c}25.06 \\
54.16 \\
64.6 \\
\end{array}$ \\
\hline & & $3.15 \mathrm{c}$ & $32.27 b$ & $47.94 a$ \\
\hline
\end{tabular}

Table 4. Effect of different concentrations of different polarity fractions of Segeratia thea (leaves) on larval mortality of the root-knot nematode, Meloidogyne incognita

\begin{tabular}{|c|c|c|c|c|}
\hline \multirow[t]{2}{*}{ Segeratia thea (leaves) } & \multirow[t]{2}{*}{ Exposure time hours } & \multicolumn{3}{|c|}{$\begin{array}{c}\% \text { mortality of juveniles at different dilutions (ppm) of plant } \\
\text { extract }\end{array}$} \\
\hline & & 10 & 100 & 1000 \\
\hline \multirow{4}{*}{ n- hexane fraction } & \multirow{4}{*}{$\begin{array}{l}24 \\
48 \\
72\end{array}$} & 1.26 & 14.63 & 41.93 \\
\hline & & 10.56 & 23.3 & 48.83 \\
\hline & & 13.6 & 27.5 & 66.7 \\
\hline & & $8.47 c$ & 21.81b & 52.48a \\
\hline \multirow{4}{*}{ Chloroform fraction } & \multirow{4}{*}{$\begin{array}{l}24 \\
48 \\
72\end{array}$} & 2.16 & 19.16 & 46.0 \\
\hline & & 12.26 & & 53.36 \\
\hline & & 16.53 & & 74.13 \\
\hline & & $10.32 \mathrm{c}$ & $25.62 \mathrm{~b}$ & 57.85a \\
\hline \multirow{4}{*}{ Ethyl acetate fraction } & \multirow{4}{*}{$\begin{array}{l}24 \\
48 \\
72\end{array}$} & 2.53 & 22.33 & 48 \\
\hline & & 14.36 & 26.13 & 54.9 \\
\hline & & 18.16 & 35.06 & 75.16 \\
\hline & & $11.68 \mathrm{c}$ & $27.84 b$ & 59.35a \\
\hline \multirow{4}{*}{ n-butanol fraction } & \multirow{4}{*}{$\begin{array}{l}24 \\
48 \\
72\end{array}$} & 1.66 & 20.3 & 46.56 \\
\hline & & 12.53 & 24.4 & 53.56 \\
\hline & & 16.4 & 33.16 & 72.00 \\
\hline & & $10.2 \mathrm{c}$ & $25.95 \mathrm{~b}$ & 57.37 a \\
\hline
\end{tabular}


Table 5. Effect of different concentrations of different polarity fractions Celtis caucasica (aerial part) on larval mortality of the root-knot nematode, Meloidogyne incognita

\begin{tabular}{|c|c|c|c|c|}
\hline \multirow[t]{2}{*}{ Celtis caucasica (aerial part) } & \multirow[t]{2}{*}{ Exposure time hours } & \multicolumn{3}{|c|}{$\begin{array}{c}\text { \% mortality of juveniles at different dilutions (ppm) of } \\
\text { plant extract }\end{array}$} \\
\hline & & 10 & 100 & 1000 \\
\hline \multirow{4}{*}{ n- hexane fraction } & \multirow{4}{*}{$\begin{array}{l}24 \\
48 \\
72\end{array}$} & 2.16 & 19 & 35.66 \\
\hline & & 9 & 29.5 & 65.36 \\
\hline & & 18.33 & 39.8 & 84.86 \\
\hline & & $9.83 c$ & 29.43b & 61.96a \\
\hline \multirow{4}{*}{ Chloroform fraction } & \multirow{4}{*}{$\begin{array}{l}24 \\
48 \\
72\end{array}$} & 2.23 & 19.2 & 40 \\
\hline & & 10.26 & 29.5 & 66.36 \\
\hline & & 20.4 & 40.83 & 86.26 \\
\hline & & $10.96 c$ & $29.84 b$ & $64.21 \mathrm{a}$ \\
\hline \multirow{4}{*}{ Ethyl acetate fraction } & \multirow{4}{*}{$\begin{array}{l}24 \\
48 \\
72\end{array}$} & 2.53 & 22.5 & 41.5 \\
\hline & & 12 & 31.73 & 68.00 \\
\hline & & 21.66 & 41.00 & 88.13 \\
\hline & & $12.06 \mathrm{c}$ & 32.01b & 65.87a \\
\hline \multirow{4}{*}{ n- butanol fraction } & \multirow{4}{*}{$\begin{array}{l}24 \\
48 \\
72\end{array}$} & 2.1 & 19.63 & 37 \\
\hline & & 9 & 30 & 65.33 \\
\hline & & 17 & 40.23 & 85.4 \\
\hline & & $9.36 \mathrm{c}$ & $29.95 b$ & $62.57 \mathrm{a}$ \\
\hline
\end{tabular}

Table 6. Effect of different concentrations of different polarity fractions of Isodon rugosus (roots) on larval mortality of the root-knot nematode, Meloidogyne incognita

\begin{tabular}{|c|c|c|c|c|}
\hline \multirow[t]{2}{*}{ Isodon rugosus (roots) } & \multirow[t]{2}{*}{ Exposure time hours } & \multicolumn{3}{|c|}{$\begin{array}{l}\text { \% mortality of juveniles at different dilutions (ppm) of } \\
\text { plant extract }\end{array}$} \\
\hline & & 10 & 100 & 1000 \\
\hline \multirow[t]{2}{*}{ n- hexane fraction } & \multirow[t]{2}{*}{$\begin{array}{l}24 \\
48 \\
72\end{array}$} & $\begin{array}{c}1.67 \\
13.33 \\
26.50\end{array}$ & $\begin{array}{c}4.7 \\
19.0 \\
36.33 \\
\end{array}$ & $\begin{array}{c}10.33 \\
21.0 \\
4\end{array}$ \\
\hline & & $13.83 \mathrm{c}$ & $20.01 b$ & 26.11a \\
\hline \multirow[t]{2}{*}{ Chloroform fraction } & \multirow[t]{2}{*}{$\begin{array}{l}24 \\
48 \\
72\end{array}$} & $\begin{array}{c}1.86 \\
14.33 \\
31.33\end{array}$ & $\begin{array}{c}5.66 \\
19 \\
42\end{array}$ & $\begin{array}{l}12.00 \\
21.66 \\
49.66\end{array}$ \\
\hline & & $15.84 c$ & $22.22 b$ & 27.77a \\
\hline \multirow[t]{2}{*}{ Ethyl acetate fraction } & \multirow[t]{2}{*}{$\begin{array}{l}24 \\
48 \\
72\end{array}$} & $\begin{array}{c}3.06 \\
14.16 \\
30 \\
\end{array}$ & $\begin{array}{c}6.08 \\
20 \\
43.83 \\
\end{array}$ & $\begin{array}{l}14 \\
25 \\
55 \\
\end{array}$ \\
\hline & & 15.74 & 23.30 & 31.33 \\
\hline \multirow[t]{2}{*}{ n- butanol fraction } & \multirow[t]{2}{*}{$\begin{array}{l}24 \\
48 \\
72\end{array}$} & $\begin{array}{c}2.63 \\
12.66 \\
26\end{array}$ & $\begin{array}{c}5.73 \\
16.66 \\
37.66\end{array}$ & $\begin{array}{c}11.13 \\
21 \\
46\end{array}$ \\
\hline & & $13.76 \mathrm{c}$ & $20.02 b$ & 26.04a \\
\hline
\end{tabular}

\section{REFERENCES}

[1] Abbasi, W. M., Ahmed, N., Zaki, J. M., Shaukat, S. S. (2008): Effect of Barleria acanthoides Vahl. on root-knot nematode infection and growth of infected okra and brinjal plants. - Pakistan Journal of Botany 40(5): 2193-2198.

[2] Abdalla, E. M., Woon, L. D., Chan, P. J., Bin, Y. H., Yul, C. H. (2008): Evaluation of various plant extracts for their nematicidal efficacies against juveniles of Meloidogyne incognita. - Journal of Asia-Pasific Entomology 11(2): 99-102.

[3] Adegbite, A. A., Adesiyan, S. O. (2005): Root extracts of plants to control root-knot Nematode on edible soybean. - World Journal of Agricultural Sciences 1(1): 18-21. 
[4] Adekunle, O. K., Akinlua, A. (2007): Nematicidal effects of Leucaena leucocephala and Gliricidia sepium extracts on Meloidogyne incognita infecting okra. - J. Agri. Sci. 52(1): 53-63.

[5] Caryrol, J. C., Djian, C., Pijarowski, I. (1989): Studies on the nematicidal properties of the culture filtrate of the nematophagous fungus Paecilomyces lilacinus. - Rev. Nematol. 12: 331-336.

[6] Chitwood, D. J. (2002): Phytochemical based strategies for nematode control. - Annual Review of Phytopathology 40: 221-249.

[7] Eisenback, J. D., Triantaphyllous, H. H. (1991): Root knot nematode Meloidogyne species and races. - In: Nickle, W. R. (ed.) Manual of Agriculture Nematology. Marcel Dekker, Inc. New York, pp. 281-286.

[8] Gommers, F. J. (1973): Nematicidal principles in Compositae. - Mededelingen Landbouwhogeschool, Wageningen, The Netherlands 17: 71-73.

[9] Javad, N., Gowmen, S. R., Ulhaq, M. I., Abdullah, K., Shahina, F. (2006): Systemic and persistent effect of neem (Azardirachta indica) formulations against root knot nematodes, Meloidogyne javanica and their storage life. - Crop Protection 26: 911-916.

[10] Knoblock, K., Weis, K., Wergent, R. (1989): Mechanism of antimicrobial activity of essential oils. - Proceedings of $37^{\text {th }}$ Annual Congress Medicine Plant Research (ACMPR'89), Braunisweig, pp. 5-9.

[11] Linford, M. B., Yap, F., Oliveira, J. M. (1938): Reduction of soil population of root-knot nematode during decomposition of organic matter. - Soil Science 45: 127-142.

[12] Muniasamy, S., Pavaraj, M., Rajan, M. K. (2010): Efficacy of the fruit extract of Citrullus colocynthis (L.) on the root-knot nematode Meloidogyne incognita infecting Vigna ungiculata (L.). - Journal of Biopesticides 3(1): 309-312.

[13] Natarajan, N., Cork, A., Boomathi, N., Pandi, R., Velavan, S., Dhaskshanamoorthy, G. (2006): Cold aqueous extracts of African marigold, Tagetes erecta for control tomato root-knot nematode. - Meloidogyne incognita Crop Protection 25: 1210-1213.

[14] Opareke, A. M., Dike, M. C., Amatobi, C. I. (2005): Field evaluation of extracts of five Nigerian species for control of post flowering insect pest of cowpea, Vignaunguiculata (L.) Walp. - Plant Protection Science 41: 14-20.

[15] Orisajo, S. B., Okeniyi, M. O., Fademi, O. A., Dongo, L. N. (2007): Nematicidal effects of water extracts of Acalypha ciliate, Jatropha gosssypifolia, Azadiractha indica and Allium ascalonicum on Meloidogyne incognita infection on cacao seedlings. - Journal of Research in Biosciences 3(3): 49-53.

[16] Pavaraj, M., Karthikairaj, K., Rajan, M. K. (2010): Effect of leaf extract of Ageratum conyzoides on the biochemical profile of blackgram Vigna mungo infected by root-knot nematode, Meloidogyne incognita. - Journal of Biopesticides 3(1): 313-316.

[17] Pavaraj, M., Bakavathiappan, G., Baskaran, S. (2012): Evaluation of some plant extracts for their nematicidal properties against root-knot nematode, Meloidogyne incognita. JBiopest 5 (Supplimentary): 106-110.

[18] Sasser, J. N., Freckman, D. W. (1987): A world perspective on nematology. The role of society. - In: Veech, J. A., Dickerson, D. W. (eds.) Vistas in Nematology. Hyattsville. Society of Nemat. pp. 7-14.

[19] Sukul, N. C. (1992): Plant antagonistic to plant parasitic nematodes. - Indian Review of Life Sciences 12: 23-52.

[20] Tsay, T. T., Wu, T. S., Lin, Y. Y. (2004): Evaluation of asteraceae plant for control of Meloidogyne incognita. - Journal of Nematology 36: 36-41.

[21] Vetrivelkalai, P., Sivakumar, M., Jonathan, E. I. (2010): Biocontrol potential of endophytic bacteria on Meloidogyne incognita and its effect on plant growth in bhendi. Journal of Biopesticides 3(2): 452-457. 